\title{
The Role of Endoscopic Surgery in the Treatment of Painful Conditions of an Aging Spine: State of the Art
}

Anthony T Yeung ${ }^{1,2,3 *}$

${ }^{1}$ University of New Mexico School of Medicine, Albuquerque, New Mexico, USA

${ }^{2}$ Society of International Intradiscal and Transforaminal Therapy, Phoenix, Arizona, USA

${ }^{3}$ Desert Institute for Spine Care, Phoenix, Arizona, USA

\begin{abstract}
Endoscopic spine surgeons bridge the major gap between fusion-oriented spinal surgeons and pain management physicians. Current surgical philosophy by traditionally trained spinal surgeons focuses on decompression with or without fusion as the "ultimate cure" for a painful spinal segment, while pain management uses spinal injections and techniques such as peripheral nerve and spinal cord neuro-modulation to relieve pain. Endoscopic surgery is the least invasive of current minimally invasive surgical options-focuses on treating the patho-anatomy of the pain generator. This represents a path between traditional open decompression with or without fusion and the more temporary techniques of pain management.
\end{abstract}

Keywords: Endoscopic surgery; Spine; Disc; Fusion techniques; Endoscopic decompression

\section{Introduction}

Common low back pain, whether affecting lifestyle or considered debilitating, will afflict most people in their lifetime due to disc degeneration from trauma or the normal aging process. Recent clinical studies demonstrate that imaging studies detecting disc degeneration even in asymptomatic patients will eventually result in the development of low back pain severe enough for patients to seek medical and surgical treatment [1-3]. Degenerative conditions in an aging spine are well documented in Cadaver cryosections by Wolfgang Rauschning and a degenerative cascade described by Pfirrmann. It is also recognized that lower back and neck pain is 3rd only after \#1 diabetes, and essentially tied with ischemic heart disease, listed as \#2, in the top 5 common health condition expenditures according to an analysis published by JAMA in 2013 (Figure 1).

\section{The patho-physiology of pain}

Why some patients develop debilitating pain or are unable to accept and tolerate common low back pain is still not completely understood, as pain is multi- factorial. Nevertheless, physicians of all backgrounds, from primary care, to non-surgical and surgical specialties all offer medical advice from their own narrow perspectives as practioners of spine care. In China, spine care is complemented by traditional nonsurgical treatments such as acupuncture with thousands of years of

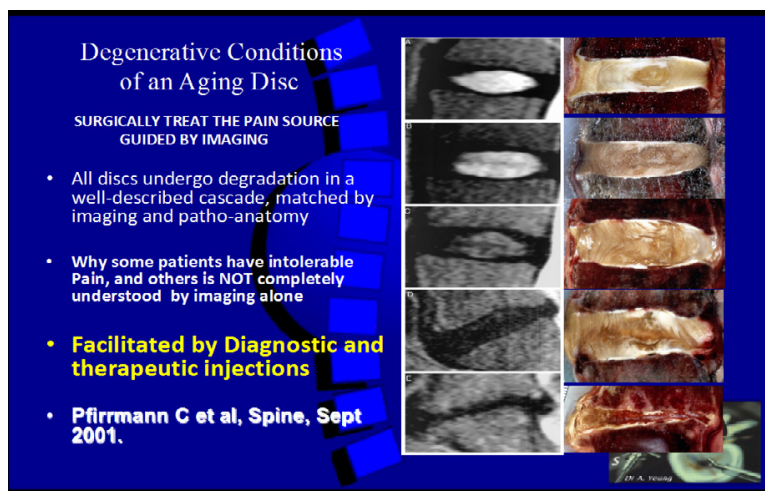

Figure 1: Degenerative conditions of an aging spine. medical history. All techniques are part of current treatment schemes and algorithms worldwide. "Modern" treatment in the United States has recently created an opioid crisis when powerful pain relieving pharmaceuticals became the easiest method to provide short-term immediate clinical relief. The procedural oriented specialties of pain management and interventional radiology has also emerged along with ever increasing minimally invasive fusion techniques.

\section{The gap between pain management and traditional surgery}

There is a major gap, however, between fusion-oriented spinal surgeons and non-surgical specialties such as pain management, physical medicine, rehabilitation, and interventional radiology among the multiple non-surgical methods treating chronic back pain and sciatica. Endoscopic spinal surgeons bridge the treatment gap. Current surgical philosophy by traditionally-trained spinal surgeons focuses on fusion as the ultimate "cure" for a painful spinal segment, justified by concepts of instability, secondary to macro or micro trauma and aging.

Pain management adopts injection procedures that ultimately depend on the natural healing process. For debilitating pain resistant to injections, peripheral nerve blocks, peripheral neuromodulation, spinal cord stimulation, or dorsal root ganglion neuromodulation is utilized, especially following "failed back surgery syndrome". Endoscopic spine surgery-the least invasive of current surgical options-offers a path between surgical fusion and the techniques of pain management.

\section{Fusion}

Fusion has also evolved throughout its modern history, with new

*Corresponding author: Anthony T. Yeung, Voluntary Professor, University of New Mexico School of Medicine, Albuquerque, New Mexico, Executive Director, Society of International Intradiscal and Transforaminal Therapy, Phoenix, Arizona and Associate Desert Institute for Spine Care, Phoenix, Arizona, USA, Tel: +1 602944-2900; E-mail: ayeung@sciatica.com

Received December 13, 2017; Accepted December 27, 2017; Published December 30, 2017

Citation: Yeung AT (2017) The Role of Endoscopic Surgery in the Treatment of Painful Conditions of an Aging Spine: State of the Art. J Neurol Disord 5: 372. doi:10.4172/2329-6895.1000372

Copyright: (c) 2017 Yeung AT. This is an open-access article distributed under the terms of the Creative Commons Attribution License, which permits unrestricted use, distribution, and reproduction in any medium, provided the original author and source are credited. 
concepts changing every few years since the pedicle screw revolution that promotes instrumented stabilization. Multiple minimally invasive fusions implants and techniques have emerged in the past 10 years, fuelled and supported by industry. Current emphasis is now on attaining sagittal alignment, another phase in the evolution of fusion techniques. Now, with the advent of MIS decompression techniques, some key opinion leaders among spine surgeons are recognizing that not all patients require instrumented fusion. Endoscopic decompression, whether translaminar or transforaminal (the least invasive) will eventually lead the way for staging procedures and offering the patient more treatment choices. Of the endoscopic techniques, the transforaminal approach is the least invasive.

\section{Pain management}

For pain management, the current focus is on injection techniques, radiofrequency rhizotomy, peripheral sensory nerve blocks, and neuromodulation of the spinal cord or dorsal root ganglion. This has been applied as a primary non-surgical procedural oriented subspecialty treatment or as a salvage technique following failed back surgery syndrome that ignores treatable patho-physiology and patho-anatomy by foraminal decompression, nerve ablation, and disc irrigation. Endoscopic surgeons promote "full endoscopic" decompression procedures as the least invasive of the surgical trends promoting minimal invasiveness in spinal surgery through translaminar or transforaminal approaches. There are now multiple subspecialty societies worldwide supporting this platform.

\section{Transforaminal endoscopic decompression and intradiscal therapy}

Patients with disc degeneration, even in asymptomatic patients have a high Risk of eventually developing low back pain, according to a prospective study assessment in Southern Chinese by the University of Hong Kong $[4,5]$. The current treatment of selective endoscopic discectomy from an extensive personal database of over 10,000 clinical cases since 1991 by Anthony Yeung has identified the disc as the common denominator in the patho-genesis of common chronic low back pain. Intradiscal therapy is the only Level I validated procedure that has under gone two large double blinded randomized studies and over 38 cohort studies. Chymopapain was eventually adopted for clinical use. It was adopted widely and utilized successfully in Korea, the United States, Europe, and Canada. Unfortunately, pharma abandoned it, and attempts to bring it back after abandonment by a multi-disciplinary group of physician users was unsuccessful due to FDA requirement roadblocks requiring new clinical studies by the FDA for bringing back an "Orphan" drug. Intradiscal therapy by minimally invasive endoscopic decompression through endoscopic disc decompression and thermal annuloplasty has subsequently evolved and has demonstrated great clinical success, with visualization and endoscopically visualized selective endoscopic discectomy and thermal annuloplasty. The disc is the main source of common low back pain, and endoscopic surgery of the disc has demonstrated great success as a major aspect and part of endoscopic intradiscal treatment (Figures 2 and 3 ).

Because endoscopic decompression of the disc is not taught in academic programs, transforaminal decompression is still misunderstood, undervalued, and underutilized even by general endoscopic surgeons. Practically, spine surgeons adopting endoscopic spine surgery, are more familiar with the anatomy of open surgical approaches, and are migrating to translaminar endoscopic approaches rather than transforaminal approaches. Transforaminal approaches, possibly more difficult for those unfamiliar to the anatomy, is still

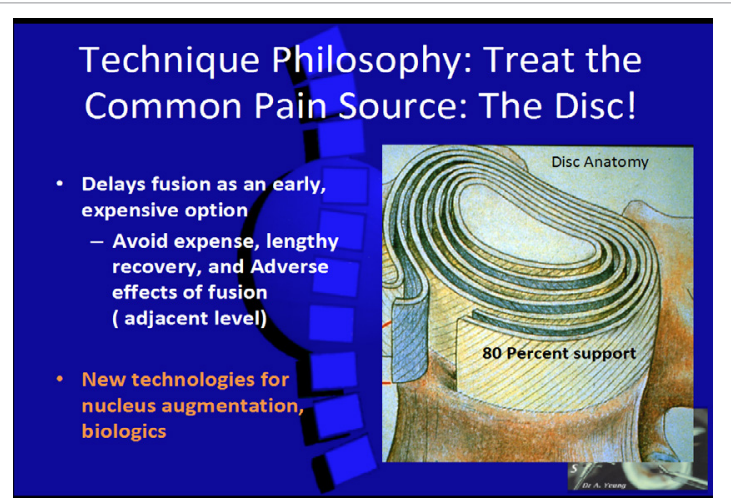

Figure 2: The disc in the anterior spinal column is responsible for $80 \%$ support of the spinal segment, and the common source of chronic low back pain.

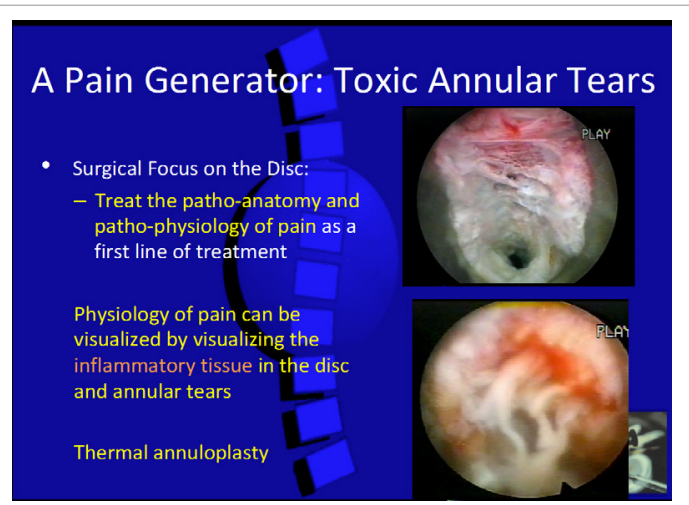

Figure 3: Toxic annular tears can be visualized endoscopically and treated with thermal annuloplasty.

safer because it is easily accomplished utilizing local anaesthesia in patients with significant co-morbidities. While endoscopic spine surgery is growing, almost all studies comparing mini open versus endoscopic trans laminar surgery or minimally open surgery demonstrate no statistically different outcomes in comparison studies, even though the endoscopic approaches have documented advantages of less surgical morbidity. Advancements in endoscopic spine surgery are being propagated mostly in private practice settings, which compete with traditionally trained surgeons.

The transforaminal approach has served to advance endoscopic spine, marketed as "full endoscopic" spine surgery when translaminar endoscopic decompression is combined with the less familiar transforaminal approach. For experienced endoscopic surgeons, with training and experience in both translaminar and transforaminal approaches, as many as $80-90 \%$ of all painful conditions can now be treated partially or fully with the endoscope. Operating under local anaesthesia, best and safest performed under local anaesthesia, enhances, and facilitates the safety and efficacy of the endoscopic platform.

\section{Health Care Reforms}

The current health care environment in the USA aims to stem the escalating cost of spine care also create roadblocks to innovation. Government and insurance payers tend to prioritize cost management over what may be the most effective treatment for a surgically stratified individual patient. The individual "surgeon factor" will also come into play. 
Payment is currently increasingly governed by guidelines developed by payers and stakeholders using evidenced-based medicine (EBM) through Level I and II validation before considering payment. Each stakeholder, therefore, focuses on level 1 and 2 EBM studies to stake out their claim for payment in the environment of shrinking reimbursement. By insisting on level 1 and 2 EBM, treatment decisions become more contorted, dictated by struggles for complex payment codes, and by playing the reimbursement game. This scenario serves as a roadblock to continued innovation for the most effective and least invasive treatment of the patient's pain dependent more on surgeon skill than expensive implants and devices.

All competing factions need new directions, with cooperation between physicians and other healthcare providers. We all need to be aware of each other's contributions as a multi-disciplinary team. Specialties need to coordinate care in cooperation with each other. Also, competing providers should consider a "warrantee" for the anticipated results of their procedures. If this concept is accepted in exchange for lifting the relentless pressure on re-imbursement, the cost of health care can be effectively curtailed while allowing continued innovation and development based on cost and value, much like how consumer products are marketed and warranted.

Our ability to respond to the individual needs of our patients will help facilitate cost containment. This will encourage innovation by preserving physician autonomy with incentive to develop new treatment through clinical and basic science research. This will allow physicians autonomy to treat patients for their individual needs with minimal interference from payers and regulators.

By focusing on the patho-anatomic source of pain through endoscopic visualization, surgical pain management using the endoscopic surgery philosophy and technique will move this subspecialty to mainstream medicine. The best method at this time is to distribute information in open access journals and training efforts. Clinical research can still be validated with traditional EBM methods of validation as long as they are also peer reviewed by qualified reviewers [6-8]. The treatment algorithm in spine is evolving and changing due to a better understanding of the patho-anatomy, well correlated with its pathophysiology. I have written about my own evolving technique in publications including a series of articles in Surgical Technology International [2,3,9-15].

\section{In vivo Visualization of Patho-anatomy}

Pain is better understood with in vivo visualization and probing of the pain generators using endoscopic transforaminal access, rather than just relying on inexact symptom diagrams. With endoscopic image correlation of the patho-anatomy of imaging studies, a new form of evidence-based medicine should be adopted. This facilitates a shared decision-making process involving the patient and surgeon, focused on a broader spectrum of surgical as well as non-surgical treatments, and not just masking the pain generator. Transforaminal endoscopic decompression, irrigation, and ablation have moved away from decisions based on imaging confirmation alone. Image abnormalities cannot, by themselves, explain the pain and disability experienced by each individual patient. Radiographic images do not always show variations in nerve supply and patho-anatomy, nor do they quantify the pain experienced by a patient. The ability to isolate and visualize "pain" generators in the foramen, treating patients under local anesthesia, and treating persistent pain by visualizing inflammation and compression of nerves, serves as the basis for transforaminal endoscopic (TFE) surgery [16-19], This has also resulted in better pre- surgical planning with more specific and defined goals in mind. There are various surgical philosophies and techniques proposed by other pioneers in endoscopic surgery, but I choose to embrace the "inside out" philosophy of TFE surgery as safe and precise in well-trained surgical hands. It provides basic access to the disc and foramen that cover a large spectrum of painful pathologies with the least surgical risk.

\section{Clinical Experience}

My own experience with the endoscopic approach for the spine began in 1991, when I took an arthroscopic spine course taught by Parviz Kambin (Philadelphia, USA) with extensive personal experience in joint arthroscopy, I began using the endoscope to explore intradiscal and foraminal anatomy. By studying Rauschning's cadaver cryoanatomy, it seemed natural to me to utilize the endoscope to examine patho-anatomy in the spine. I quickly decided to visualize the epidural space through the foramen with the "inside out" technique to obtain confirmation that the spinal nerves were decompressed. Visualized use of Holmium;Yag laser for fine bone and soft tissue dissection was also demonstrated to be a valuable surgical tool (Figure 4).

\section{Laser as a surgical tool}

In 1991, the laser was introduced for the lumbar spine, and I became a fan of laser as a surgical tool under endoscopic visualization, because it had the tissue effect of decreasing bleeding, ablating soft tissue as well as bone. The small working surgical portal made it an effective surgical tool through an endoscope directed to the disc and foramen ad epidural space through a $6 \mathrm{~mm}$ inner diameter surgical cannula and an endoscope with a $2.8 \mathrm{~mm}$ to $4.1 \mathrm{~mm}$ working channel endoscope [20-24].

\section{The development of the Yeung Endoscopic Spine System (YESS)}

This experience caused me to develop my own endoscopic system in partnership with Richard Wolf's surgical instrument company. In 1997, the Yeung Endoscopic Spine System (YESS) was cleared by the US Food and Drug administration (FDA) and marketed in the USA (Figure 5).

I have not deviated from this surgical philosophy since then, except to evolve the technique, aided by the recognition of spinal patho-anatomy. I have customized my surgical instrumentation to facilitate decompression, ablation, and irrigation of the pain generator. The patho-anatomy is identified by the endoscope, and probing, decompression, ablation or transection treats the effect of surgery under local anaesthesia.

My original endoscope design is still the state of the art configuration for intradiscal therapy, where the degenerative process begins. It has been copied and reproduced to some extent in designs

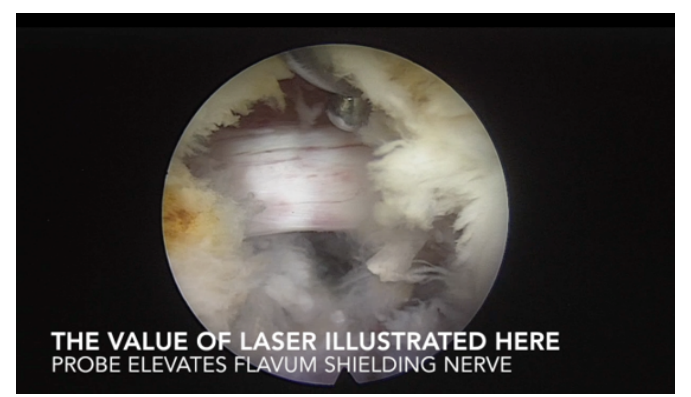

Figure 4: The traversing nerve is visualized following foraminoplasty and laser ablation of the ligamentum flavum shielding the nerve at the inlet of the foramen. 
Citation: Yeung AT (2017) The Role of Endoscopic Surgery in the Treatment of Painful Conditions of an Aging Spine: State of the Art. J Neurol Disord 5: 372. doi:10.4172/2329-6895.1000372

Page 4 of 5

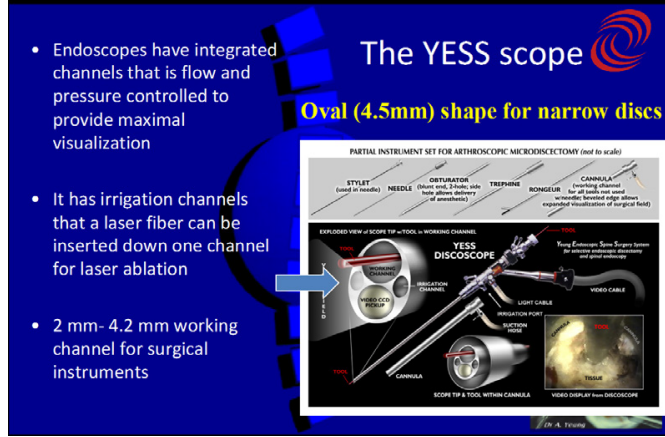

Figure 5: YESS scope designed for intradiscal therapy and epidural exploration.

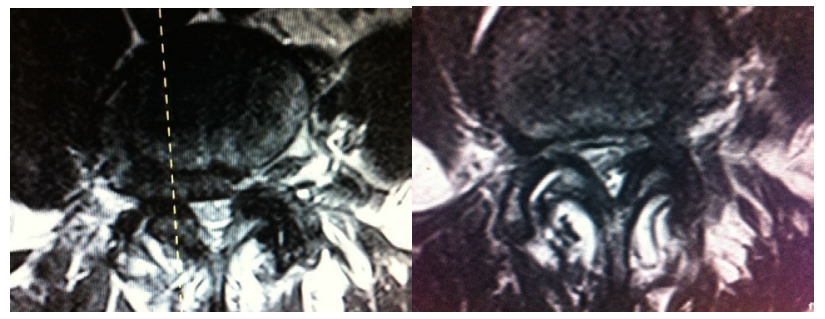

Anthony Yeung's Pre-op MRI Anthony Yeung's Post-op MRI

Figure 6: Endoscopic foraminal decompression of A. Yeung's multimodal HNP.

of other endoscope manufacturers. As the procedure evolved, different scope sizes, designs and working channels have been augmented by better endoscopic instrumentation and modified approach techniques. Instruments have also evolved to make endoscopic spinal surgery easier, safer, and more effective as a staple of the surgical treatment of the future [25-27].

\section{Personal testimony}

I end with a personal account of my own spinal condition, a multimodal HNP that was intermittently symptomatic for 5 years. The symptoms deteriorated rapidly over the final three months before I opted for surgical intervention only after I experienced symptoms of persistent and progressive pain, numbness, and weakness. An epidural transforaminal steroid block provided relief for less than 24 hours. I then obtained an MRI that demonstrated the large multi-modal HNP. In retrospect, I should have had confirming imaging and endoscopic foraminal surgery months earlier. Although I initially adhered to my career long focus on conservative treatment first, then treating pain generators with transforaminal endoscopic surgery, I conclude that waiting for the development of a neurologic deficit was not efficient, not necessary, nor cost effective, if it affects a patient's ability to work or function. I also conclude that the use of transforaminal diagnostic and therapeutic injections could also help stratify patients seeking endoscopic transforaminal decompression for relief of their chronic back pain and sciatica. I underwent the surgery that I developed using only local anaesthesia and NO sedation. The surgery was performed by my son, a traditionally fellowship trained spinal surgeon, Christopher Yeung, Chris was trained by me in endoscopic spine surgery while he was an associate at our spine practice, Desert Institute for Spine Care (Figures 6a and 6b).

\section{Discussion and Conclusion}

With the current information treating visualized patho-anatomy, earlier surgical intervention can and would have provided relief earlier in the disease process, facilitating surgical recovery and ultimately improving long-term results. Waiting for objective neurologic findings of nerve deficits can hinder rapid clinical recovery. With the current state of the art development of endoscopic surgery, we are destined to improve spine care by moving away from fusion as a first line surgical treatment because of the effective techniques available in the endoscopic platform. To attain this level of surgical expertise, appropriate training and ongoing clinical experience is paramount. The efficacy of the technique is surgeon dependent. This is not a "see one, do one, teach one" procedure. Endoscopic transforaminal surgery has both a steep or shallow learning curve dependent on the "surgeon factor", but the learning curve is helped greatly by scrubbing with an experienced surgical mentor. Having the experienced surgeon scrubbing in will shorten the surgical learning process, and both technical and clinical experience helps tremendously. With the patient awake, however, patients seeking this type of surgery may have some anxiety when the endoscopic surgeon's patient agrees to have a student scrub in.

\section{References}

1. Samartis D (2010) Disc degeneration in asymptomatic patients increases risk of low: back pain, a prospectively assessment of Southern Chinese.

2. Yeung AT, Gore S (2011) In-vivo endoscopic visualization of patho-anatomy in symptomatic degenerative conditions of the lumbar spine II: Intradiscal, foraminal, and central canal decompression. Surg Technol Int 21: 299-319.

3. Yeung AT, Yeung CA (2011) Posterolateral endoscopic lumbar diskectomy. In Advanced Reconstruction. Spine pp: 611-624.

4. Yeung AT, Yeung CA, Meredith CC (2011) Endoscopic surgical pain management in the aging spine. In: The comprehensive treatment of the aging spine: Minimally invasive and advanced techniques. Philadelphia, Elsevier Saunders, The Netherlands.

5. Yeung AT, Yeung CA (2011) Posterolateral endoscopic lumbar discectomy. In Rothman-Simeone. The Spine 1041-1050.

6. Yeung AT (2014) Foreword, percutaneous and endoscopic MIS (Special issue) Special edition. Int J Spine Surg 8: 14

7. Yeung, AT, Gore S (2014) Endoscopically guided foraminal and dorsa rhizotomy for chronic axial back pain based on cadaver and endoscopically visualized anatomic study. Int J Spine Surg; 23.

8. Yeung AT, Gore S (2014) Endoscopic foraminal decompression for failed back surgery syndrome under local anesthesia. Int J Spine Surg 8: 22.

9. Gore S, Yeung AT (2014) The "inside out" transforaminal technique to treat lumbar spinal pain in an awake and aware patient under local anesthesia: Results and a review of the literature. Int J Spine Surg 8: 28.

10. Yeung AT, Gore S (2015) Twenty-three years of experience with percutaneous transforaminal spine surgery: It's evolution, the painful conditions treated, results, personal thoughts, and a review of the evolving literature. Surg Tech Int: 25 .

11. Yeung AT (2015) Moving away from fusion by treating the pain generator: The secrets of an endoscopic master. J Spine 4: e121.

12. Weiner R, Yeung AT, Perryman L, Carlos MG, Benjamin S (2016) Treatment of FBSS low back pain with a novel percutaneous DRG wireless stimulator: A pilot and feasibility study. Pain Medicine 17: 1911-1916.

13. Yeung CA, Field JS, Yeung AT (2015) Endoscopic foraminal approach to the lumbar spine. In: Surgical approaches to the spine 52: 365-378.

14. Yeung AT (2017) Intradiscal therapy and nucleus augmentation as a surgical technique for the treatment of common and chronic low back pain. J Spine Neurosurg 6.

15. Yeung AT (2016) Endoscopic decompression, foraminalplasty and dorsa rhizotomy for foraminal stenosis and lumbar spondylosis: A hybrid procedure in lieu of fusion. J Neurol Disord 4: 322.

16. Yeung AT (2016) Intradiscal therapy and transforaminal endoscopic decompression: Opportunities and challenges for the future. JNeurol Disord 4: 303.

17. Yeung AT (2017) Delivery of spine care under health care reform in the United States. J Spine 6: 372 
Citation: Yeung AT (2017) The Role of Endoscopic Surgery in the Treatment of Painful Conditions of an Aging Spine: State of the Art. J Neurol Disord 5: 372. doi:10.4172/2329-6895.1000372

Page 5 of 5

18. Yeung AT (2017) Transforaminal endoscopic decompression for painful degenerative conditions of the lumbar spine: A review of one surgeon's experience with over 10,000 cases since 1991. J Spine Neurosurg 6: 266.

19. Anthony TY, Christopher AY (2017) Selective endoscopic lumbar discectomy $\left(\mathrm{SED}^{\mathrm{TM}}\right.$ ) and thermal annuloplasty for discogenic back pain, disc herniations and sciatica in high performance athletes and physically active patients. Sports Injr Med JSIMD: 110.

20. Yeung AT (2017) Robotics in the MIS Spine Surgery Arena: A new role to advance the adoption of endoscopic surgery as the least invasive spine surgery procedure. J Spine 6: 374.

21. Yeung A, Yeung CA (2017) Endoscopic identification and treating the pain generators in the lumbar spine that escape detection by traditional imaging studies. J Spine 6: 369.

22. Anthony T Yeung (2017) Failed back surgery syndrome: Endoscopic documentation of common causes by visualization of painful patho-anatomy in the hidden zone of the axilla containing the dorsal root ganglion and salvage treatment of neuropathic pain with DRG neuromodulation. SF J Neuro Sci 1: 1

23. Yeung AT (2017) A short note on minimally invasive lumbar spine surgery. J Spine 6: e127.

24. Zhang X, Du J, Yeung AT (2017) Development of percutaneous endoscopic lumbar discectomy (PELD) technology in China. J Spine 6: 374

25. Yeung AT (2015) Short Commentary for an open access Journal in BiBA News

26. Yeung AT, Yeung CA, Salari N, Field J, Navratil J, et al. (2017) Lessons learned using local anesthesia for minimally invasive endoscopic spine surgery. J Spine 6: 377.

27. Yeung AT (2017) In-vivo endoscopic visualization of pain generators in the lumbar spine. J Spine 6: 385 